\title{
Desarrollo de la Competencia de Trabajo en Equipo en la Asignatura de Dirección de Producción y Operaciones
}

\author{
Sofía Estelles-Miguel ${ }^{\mathrm{a}}$, José Miguel Albarracín Guillem ${ }^{\mathrm{b}}$, Marta Elena Palmer Gato ${ }^{\mathrm{c}}$ y \\ Marta Peris-Ortiz ${ }^{\text {d }}$ \\ a,b,c Facultad de Administración y Dirección de Empresas, Departamento de Ortanización de \\ Empresas-Universitat Politècnica de València soesmi@omp.upv.es; jmalbarr@omp.upv.es; \\ marpalga@omp.upv.es y ${ }^{\mathrm{d}}$ Escuela Técnica Superior de Ingenieros Industriales de Valencia \\ mperis@doe.upv.es. Todos del Departamento de Organización de Empresas de la Universitat \\ Politècnica de València.
}

\begin{abstract}
Teamwork has become one of the demands in the labor market. This is due to the changes, the incorporation of technologies and the new processes that make the tasks become increasingly complex and in many cases it is not possible to obtain and individual resolution of them. Therefore, in many organizations are trying to create a culture of collaboration as the best strategy for problem solving. However the education that should promote cooperation and teamwork currently still promotes competitiveness. Based on all this, a teamwork experience has been developed in the Operations Management subject and this paper presents the results of the same.
\end{abstract}

Keywords:competences, skills, teamwork, cooperative and collaborative education.

\footnotetext{
Resumen

El trabajo en equipo se ha convertido en una exigencia en el mercado laboral. Esto es debido a que los cambios, la incorporación de tecnologías y los nuevos procesos dentro de las organizaciones hacen que las tareas sean cada vez más complejas y en muchas ocasiones no es posible realizar una resolución individual de las mismas. Por todo esto, en la mayoría de las organizaciones se pretende crear una cultura de colaboración, como la mejor estrategia para la resolución de problemas. Sin embargo, la educación que debería promover la cooperación y el trabajo en equipo, actualmente todavía promueve la competitividad. En base a todo esto, se ha desarrollado una experiencia de trabajo en equipo en la asignatura de Dirección de Producción y Operaciones y el presente trabajo presenta los resultados de la misma.
}

Palabras clave: competencias, habilidades, trabajo en equipo, educación cooperativa y colaborativa. 


\section{Introducción}

Algunos estudios demuestran que los niños que trabajan de forma cooperativa en el colegio, consiguen mejores resultados académicos y se comprometen en actividades sociales más frecuentemente (Ricard, 2010). Sin embargo en la mayoría de centros educativos se sigue incentivando, algunas veces sin ser conscientes de ello, la competitividad. Pero sabemos que es más efectivo trabajar en equipo que hacerlo de forma individual. Algunas razones para ello son (Gómez Pereira, 2016):

- Porque se complementan las habilidades y los talentos.

- Porque se terminan más rápido las tareas.

- Porque confías y te vuelves confiable.

- Porque aumenta el sentido de pertenencia.

- Porque es gratificante para todos.

- Porque no estarás solo.

- Porque únicamente así se alcanzan las metas.

- Porque de esa manera aprendes más de ti.

- Porque puedes resolver mejor los conflictos y encontrar nuevas ideas.

- Porque es más divertido.

En la actualidad la mayoría de las organizaciones está fomentando una manera de trabajar más cooperativa y colaborativa. La complejidad del mundo laboral actual y la innovación generan situaciones en las que se requieren de distintas habilidades, altos niveles de conocimiento, adaptabilidad y rápidas respuestas. Y sólo a través del trabajo en equipo es donde se puede obtener todas estas características juntas (Kozlowski e Illgen, 2006).

Por todo ello, cada vez se utiliza más el trabajo en equipo en la mayoría de empresas y organizaciones (Alcover et al, 2004; Aritzeta y Balluerka, 2006; Ellis et al, 2005; Gruenfeld et al, 1996; Hollenbeck et al, 2004; Janz et al, 1996; Kozlowski e Illgen, 2006; Miklavic et al, 2007; Park et al, 2005, Yeh et al, 2006 y Torrelles et al, 2011).

El trabajo en equipo precisa de la colaboración y cooperación de distintos miembros de las empresas con diferentes habilidades, de esta forma se mejora la participación, la comunicación y se incrementa la calidad (Cohen y Bailey, 1997; Ellis et al, 2005 y Park et al, 2005).

Hollenbeck et al (2004) dice que el trabajo en equipo ha ido incrementándose notablemente desde los años 80 del pasado siglo en las empresas. Pero se ha introducido si cabe más desde la década de los 90 del siglo XX (Mazany et al, 1995; McDermott, 1999 y Park et al, 2005).

Los equipos se consideran entidades complejas, dinámicas, adaptables e integradas en un sistema de distintos niveles. Estos sistemas deben de tener en cuenta por un lado al individuo, por otro al equipo y finalmente a la organización (Torrelles et al, 2011). El trabajo en equipo necesita de la movilización de recursos tanto propios como externos. Por otro lado también necesita que el individuo disponga de ciertos conocimientos, habilidades $\mathrm{y}$ aptitudes para adaptarse al equipo y a las situaciones que se van a ir presentando. $\mathrm{Si}$

(cc) EY-NC-ND 2017, Universitat Politècnica de València 
alguno de los miembros del equipo no dispone de los elementos imprescindibles para el trabajo en equipo, puede hacer que el equipo fracase y no se obtengan los resultados esperados. Por todo esto, hay que generar dinámicas que ayuden a las personas a prepararse para el trabajo en equipo.

Trabajar en equipo requiere de una serie de habilidades como:

- El dar y recibir realimentación, es decir, saber que estamos haciendo bien y mal y saber corregirlo, siempre desde el punto de vista de la mejora continua y no de la crítica.

- La adaptación, es decir, saber adaptarnos a las distintas situaciones y a las distintas necesidades del grupo y cubrir aquellos espacios que queden vacíos o flojos.

- La gestión del tiempo. En el trabajo en grupo es tan importante el saber gestionar el tiempo del que disponemos para la realización de la tarea como el resto de habilidades.

- La escucha activa, saber escuchar las informaciones que nos aportan los demás y no sólo querer que se oiga nuestra opinión.

- La colaboración, que todos somos parte del equipo y todos debemos aportar nuestros granos de arena y participar de la mejor manera que sepamos y que podamos.

- Optimismo, apoyar el equipo incluso cuando las situaciones son adversas. La gente quizás en esos momentos es cuando más apoyo necesita.

Con todo esto en mente y pretendiendo que los alumnos de Facultad de Administración y Dirección de Empresas (FADE) de la Universitat Politècnica de València (UPV) salgan preparados para el trabajo en equipo los profesores de la asignatura de Dirección de Producción y Operaciones (DPO) han desarrollado el presente trabajo. Con este trabajo se pretende desarrollar la habilidad del trabajo en grupo a través de dinámicas y la realización de trabajos en grupo en parte de la asignatura.

\section{Desarrollo de la Metodología en la Asignatura}

Cuanto más se aprende es cuanto más se parece el contexto del aprendizaje a la vida real. Es decir, si la simulación o práctica que estoy realizando, se parece a lo que me voy a encontrar en la realidad, el alumno aprende más. Por esto, es importante que los estudiantes puedan aplicar lo que aprenden, o por lo menos puedan verle una aplicabilidad, en dicho caso será mejor entendido. La implantación de metodologías activas en el aula permite la formación en competencias del alumnado (González y Wagenaar, 2003 y Estelles-Miguel et al, 2013).

En la presente asignatura se propuso un proyecto a los alumnos en el cual tenían que trabajar en grupos para la resolución del mismo, además el trabajo debía realizarse de forma cooperativa y colaborativa para poder alcanzar un resultado no sólo lógico sino coherente.

Los objetivos al plantear este trabajo a los alumnos fueron:

- Potenciar la autonomía de los alumnos, el trabajo en equipo, el pensamiento reflexivo y crítico. 
- Que los alumnos desarrollen destrezas profesionales, que adquieran capacidades para identificar, plantear y resolver problemas relacionados con los contenidos prácticos de la asignatura.

Aunque algunos autores dicen que es mejor que los grupos sean formados por alumnos con diferentes habilidades y características, y normalmente para ello deben ser seleccionados por los profesores, en el presente caso, se les dejo a los propios alumnos seleccionar los participantes del grupo de trabajo.

Los grupos de trabajo debían ser de cinco componentes, existían 5 roles distintos que luego comentaremos y entre ellos también debían seleccionar que rol ocuparía cada uno de ellos.

En la primera parte del ejercicio y antes de que definan sus roles, se les entrega un problema claramente definido. En el que se indica que, dos alumnos van a crear una empresa y se les dice cual va a ser su proceso de fabricación (incluyendo máquinas y tiempos de las mismas), cuáles van a ser sus productos finales (incluyendo ingredientes, cantidades, etc.). En este caso se supone que el proceso de fabricación es artesanal, es decir, disponen de un horno casero y las empanadillas son fabricadas por los propios alumnos. Con estos datos tienen que calcular tiempos de procesado de un lote y cuantos lotes pueden realizar en un turno de trabajo. Para ello, tendrán que ver cuál es el cuello de botella del proceso aunque no se les indica este dato. Una vez han realizado esta primera parte y la han entregado se corregirá en la pizarra para que vean sus errores.

Para la segunda parte del trabajo, los alumnos deberán asignar los roles, éstos son los siguientes:

- Director: Es el responsable de coordinar el grupo de trabajo, de gestionar los tiempos, de realizar aquellas tareas que por cualquier motivo no pueda realizar otro. Es el encargado del portafolio y de su presentación. Y finalmente deberá realizar una valoración del trabajo de sus compañeros de grupo.

- Responsable de Producto: Es el especialista en este tema, deberá preparar una presentación sobre esta parte, y será el que más conozca este tema y apoye a su grupo durante la realización de esta parte del trabajo.

- Responsable de Proceso: Es el especialista en este tema, deberá preparar una presentación sobre esta parte, y será el que más conozca este tema y apoye a su grupo durante la realización de esta parte del trabajo.

- Responsable de Distribución en Planta: Es el especialista en este tema, deberá preparar una presentación sobre esta parte, y será el que más conozca este tema y apoye a su grupo durante la realización de esta parte del trabajo.

- Responsable de Localización: Es el especialista en este tema, deberá preparar una presentación sobre esta parte, y será el que más conozca este tema y apoye a su grupo durante la realización de esta parte del trabajo.

Una vez repartidas las tareas empezarán a trabajar en grupo en el problema y en cada una de estas partes. El proyecto consiste en crear una empresa de fabricación de empanadillas. Al comenzar con cada una de las partes, los especialistas del tema de los distintos grupos saldrán y expondrán el tema a toda la clase. A partir de ese momento, se pondrán a trabajar

(cc) EY-NC-ND 2017, Universitat Politècnica de València 
cada uno en su grupo, y dependiendo de la tarea tendrán una o dos semanas para cada una de las partes. Las partes son las siguientes:

- El primer tema es diseño de producto. En el mismo los grupos deberán definir que producto van a fabricar todos harán empanadillas, pero deben decidir: tamaño, sabores, materias primas, forma, variedades, etc. Además de la información que se les entrega pueden consultar toda la información que necesiten en internet, y tienen que seguir los pasos de diseño de producto que habrán visto con su responsable.

- En segundo lugar se llega a diseño de proceso. Todos los grupos deberán definir cómo van a fabricar las empanadillas que han decidido fabricar en el primer paso, las decisiones que hayan tomado en el diseño de producto les afectarán en este paso. Deberán elegir máquinas, herramientas, cuantas personas van a trabajar, turnos, etc. Deberán seguir todos los pasos que su responsable les habrá indicado. Al igual que en el caso anterior los profesores les pasaremos información sobre máquinas, pero pueden buscar otras.

- Distribución en Planta, una vez han decidido que máquinas van a comprar deberán ver como las van a instalar en su proceso productivo y como les afecta. Las decisiones que hayan tomado en el paso anterior afectarán en este paso.

- Finalmente tendrán que decidir la localización de sus instalaciones, que no será la misma si van a tener una fábrica que suministre a un gran supermercado que si tienen un horno tradicional.

Los alumnos deberán tener en cuenta que, cada decisión tomada influye en los siguientes pasos a realizar y no existe una única solución buena. Cada grupo tendrá una empresa distinta, con unos productos distintos, unos procesos distintos, una distribución en planta distinta y una localización única y distinta. Las decisiones deben ser coherentes unas con otras para que la solución sea lógica y razonable. Para ello tendrán que utilizar además de los cálculos, la información que los profesores les aportan y la información que obtengan por distintos medios una herramienta muy necesaria es el sentido común. Todo ello se llevará en un portafolio. No existe un modelo único de portafolio, al existir distintas modalidades en función de los objetivos que se persiguen con su desarrollo (EstellesMiguel et al, 2013).

Si algún día un responsable de un grupo no puede acudir, tendrá que ser sustituido en sus labores por el director del grupo, es decir, el show no puede parar.

\section{Resultados obtenidos}

En los trabajos en equipo a través de las interdependencias entre sus miembros se revelan las distintas personalidades de los participantes del equipo. Cada uno de nosotros tiene unas características en su personalidad, parte de ellas debida a la genética y otras fruto de la educación y las experiencias. Cuando trabajamos en equipo la personalidad de cada uno de los miembros del equipo se revela en función de los roles que cada uno de ellos asumen. 
El desarrollo de trabajos supone un complemento adecuado a las clases teóricas y prácticas, ya que con ello no sólo se transmiten conocimientos al alumno sino también la posibilidad de desarrollar destrezas y habilidades (Flórez y Albelda, 2012).

En el trabajo en equipo realizado durante el curso 2016/2017 en la asignatura de Dirección de Operaciones se ha observado que nuestros alumnos no están acostumbrados al trabajo en equipo. Sino más bien a un trabajo seriado, en el que se reparten cada uno una parte del trabajo, para posteriormente juntarlas y presentarlas. En el presente caso, ese método no les ha servido, por lo que se han visto obligados a trabajar de otra forma y adaptarse al trabajo cooperativo y colaborativo. Es más, se han visto obligados a realizar sesiones de tormentas de ideas, en las que todos colaboraban y posteriormente se seleccionaban las mejores ideas. Con ello, se han dado cuenta de que cada persona puede aportar una forma distinta de ver las cosas y que eso es enriquecedor.

Se paso un cuestionario con escala Likert ( 1 a 3 ) siendo 1 nada de acuerdo y 3 totalmente de acuerdo sobre el trabajo en equipo con las siguientes preguntas:

- Consideras que el trabajo en equipo :

- Fomenta la capacidad de organización y planificación del trabajo.

- Facilita las relaciones con tus compañeros y con los profesores.

- Ayuda a potenciar la autonomía en el aprendizaje.

- Ayuda a reflexionar sobre los problemas encontrados y los resultados.

- Favorece la motivación.

- Ayuda a aplicar conocimientos.

- Ayuda en la gestión del tiempo.

- Las respuestas obtenidas fueron:

- Fomenta la capacidad de organización y planificación del trabajo: 3\% nada de acuerdo, $33 \%$ de acuerdo y $64 \%$ completamente de acuerdo.

- Facilita las relaciones con tus compañeros y con los profesores: $10 \%$ nada de acuerdo, 39\% de acuerdo y 51\% completamente de acuerdo.

- Ayuda a potenciar la autonomía en el aprendizaje: 5\% nada de acuerdo, $26 \%$ de acuerdo y $79 \%$ completamente de acuerdo.

- Ayuda a reflexionar sobre los problemas encontrados y los resultados: $48 \%$ de acuerdo y $52 \%$ completamente de acuerdo.

○ Favorece la motivación: 7\% nada de acuerdo, 26\% de acuerdo y $67 \%$ completamente de acuerdo.

○ Ayuda a aplicar conocimientos: $23 \%$ de acuerdo y $77 \%$ completamente de acuerdo.

- Ayuda en la gestión del tiempo: 3\% nada de acuerdo, $36 \%$ de acuerdo y $61 \%$ completamente de acuerdo.

Los profesores consideran que incrementa su carga de trabajo pero que es adecuado para mejorar el aprendizaje y las habilidades de los alumnos.

(cc) EY-NC-ND 2017, Universitat Politècnica de València 


\section{Conclusiones}

El presente trabajo presenta la experiencia realizada en la aplicación del trabajo en equipo desarrollada en la asignatura de Dirección de Operaciones en el primer semestre de segundo curso en el Grado de Administración y Dirección de Empresas en la Universitat Politècnica de València. En estos momentos todavía son escasos los datos para poder valorarla.

En este trabajo se ha comprobado que la realización de trabajo en grupo potencia algunas habilidades como: la autonomía en el aprendizaje (según los alumnos lo que más), seguida de que es útil para llevar los conocimientos a la práctica, favorece la motivación de los alumnos, fomenta la capacidad de organización y planificación del trabajo, aprenden a gestionar su tiempo. Habilidades todas ellas muy necesarias para el desarrollo de la vida laboral.

Los alumnos dijeron en sus comentarios que se deberían realizar esté tipo de prácticas en más asignaturas. Si bien es cierto que les consume mucho tiempo y si se hicieran en todas las asignaturas no saben si serían capaces de llevarlos a buen término.

Según Thomas (2000), el trabajo en proyectos que se ha utilizado en el presente caso para la aplicación del trabajo en equipo, es relativamente difícil de planificar y de llevar a cabo. Todos los profesores participantes en esta experiencia en la asignatura de Dirección de Operaciones encontraron alguna dificultad a la hora de llevarlo a cabo, las cuálesse fueron superando. También el diseñar una actividad de aprendizaje basada en el trabajo colaborativo y cooperativo supone para el docente una importante carga de trabajo, además de un reto diario en el aula.

La mayor carga de trabajo para los profesores fue la elaboración del proyecto en sí, y posteriormente las actividades a realizar durante las clases en las que se trabajaba en equipo y posteriormente la corrección de dicho trabajo. Teniendo en cuenta de que no existe una única solución correcta. Qué todas las soluciones son válidas siempre que sean lógicas y consecuentes y que hayan tenido en cuenta tanto los parámetros indicados en el trabajo como las decisiones tomadas en cada uno de los pasos previos. A todo ello, hay que sumar la incertidumbre y la falta de control en algunos casos al realizar estas tareas "en vivo" cosa que no ocurre en las clases magistrales.

Existen evidencias de que algunos estudiantes encuentran dificultades a la hora de auto dirigirse, especialmente en proyectos complejos, con la gestión del tiempo y de proyectos (Thomas, 2000). Nosotros diríamos no sólo de auto dirigirse como personas, sino de trabajar en equipo y coordinar un equipo de trabajo. Pero con esto aprenden o mejoran una de las habilidades que las empresas demandan la del trabajo en equipo. Además, es mejor que salgan de la universidad enseñados, que se hayan enfrentado a un reto de este tipo, antes de hacerlo por primera vez en una empresa. Todo esto puede solucionarse siempre que haya ganas de hacerlo, actitud y un apoyo (que no dirección) por parte de los profesores. Los profesores deben actuar de facilitadores.

En la experiencia realizada se ha visto que con este tipo de trabajos los alumnos aprenden haciendo y lo hacen con problemas reales. Además interiorizan las habilidades de trabajo 
colaborativo, cooperativo y trabajo en equipo, al tener que trabajar con otras personas y que su trabajo influya en el de los demás. Otras de las competencias que desarrollan es la gestión del tiempo (como existe un tiempo limitado para cada tarea se tienen que gestionar), la responsabilidad individual, la capacidad reflexiva (al influir unas decisiones en las siguientes), a manejar las relaciones interpersonales (que no todos somos ni pensamos igual y que hay que aceptar a los demás). Todas esta habilidades y capacidades les servirán en su vida profesional, en ocasiones incluso más que sus conocimientos académicos.

Habrá que corregir los problemas que se han detectado. Aunque habría que añadir que aún con todos los fallos encontrados los autores del presente trabajo entienden que ha sido una experiencia completamente gratificante para ellos y para sus estudiantes.

\section{Referencias}

ALCOVER, C, FIL, F y BARRASA A. (2004). "Team Learning: Adaptation of learning activities scales in a Spanish sample" en Psicothema, vol. 16, No 3, p. 378-383.

ARITZETA, A y BALLUERCA, N. (2006). "Cooperation, competition and goal interdependence in work teams : A multilevel approach” en Psicothema, vol. 18, No 4, pp. 757-765.

COHEN, S y BAILEY, D. (1997). "What makes teams work : Group effectiveness research from the shop floor to the executive suite" en Journal of Management, vol. 23, No 3, pp. 239-290.

ELLIS, A, BELL, B, PLOYHART, R, HOLLENBECK, J e ILLGEN, D. (2005). “An evaluation of generic teamwork skills training with action teams: Effects on congnitive and skill-based outcomes" en Personnel Psycology, vol. 58, pp. 641-672.

ESTELLÉS-MIGUEL, S, BARBERA RIBERA, T y DEMA PÉREZ, CM. (2013). ). “Aplicación del portafolio grupal en la asignatura de Dirección de Producción y Logística: Resultados de una prueba poiloto", No4, pp. 124-138.

FLÓREZ R. y ALBELDA, E. (2012). "Una experiencia de trabajo en equipo con soporte documental para la formación de competencias en contabilidad financiera" en Revista Educade, $\mathrm{N}^{\mathrm{o}} 3$, pp. 67-94.

GÓMEZ PEREIRA, Belén (2016). "10 Razones para Trabajar en Equipo". https://www.entrepreneur.com/article/267218 Publicado: 04 de julio de 2016. [Consulta: 14 de marzo de 2017].

GONZÁLEZ, J. y WAGENAAR, R (EDS) (2003). "Tuning Educational Structures in Europe. Informe Final. Fase Uno”. Bilbao : Universidad de Deusto y Universidad de Groningen.

GRUENFELD, D, MANNIX,E, WILLIANS, K y NEALE, M. (1996). “Group Composition and Decision Making: How Member Familiarity and Information Distribution Affect Process and Performance.” en Organizational Behaviour and Human Decision Processes, vol. 67, No 1, pp. 1-15.

HOLLENBECK, J, DEREUE, D. y FUZZO, R. (2004). "Bridging the gap between I/O research and HR practice: Improving team composition, team training and team task design" en Resource Management, vol. 43, pp. 353-366.

JANZ, B, COLQUITT, J y NOE R. (1997). "Knowledge Yorker Team Effectiveness : The role of autonomy, interdependence, team, development and contextual support variabless" en Personnel Psycology, vol. 50, pp. 877-904.

KOZLOWSKI, S.W. y ILLGEN, D.R.. (2006). "Enhancing the Effectiveness of Works Groups and Teams" en Psychological Science in the Public Interest, vol. 7, № 3, pp. 77-124.

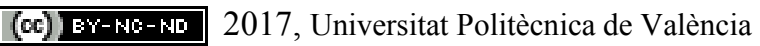


MAZANY, P, FRANCIS,S y SUMICH, P. (1995). "Evaluating the Effectiveness of an Outdoor Workshop for Team Building in an MBA Programme" en Journal of Management Development, vol. $14, \mathrm{~N}^{\mathrm{o}} 3$, pp. $50-68$.

McDERMOTT, R. (1999). "Learning Across Teams: The Role of Communities of Practice in Team Organizations" en Knowledge Management Review, pp. 1-8.

MIKLAVCIC SAMANSKI, M, KOLENC, I y MARKIC, M.. (2007). "Teamwork and refining group structures" en Team Performance Management, vol. 13, No 3/4, pp. 102-116.

PARK, S, HENKIN, A. y EGLEY, R. (2005). "Teacher team commitment, teamwork and trust: Exploring associations" en Journal of Educational Administration, vol. 43, No 5, pp.462-479.

RICARD, Matthieu (2010). "Educación: Promueve la cooperación, no la competitividad". http://www.matthieuricard.org/es/blog/posts/educacion-promueve-la-cooperacion-no-lacompetitividad [Consulta: 14 de marzo de 2017].

THOMAS, J. (2000). "A Review of Research on Project-Based Learning". http://www.autodesk.com/foundation Publicado en Marzo de 2000. [Consulta 17 de marzo de 2017.

TORREllES, C, COIDURAS, J, ISUS, S, CARRERA, X, PARÍS, G. Y CELA, J.M. (2011). "Competencia de Trabajo en Equipo: Definición y Categorización" en Revista del Currículum y $\begin{array}{llllll}\text { Formación del } & \text { Profesorado } & \text { Vol. } & \text { 15, } 329-345 .\end{array}$ http://www.ugr.es/local/recfpro/rev153COL8.pdf

YEH, E, SMITH,C, JENNINGS, C y CASTRO, N. (2006). "Team Building : a3-Dimensional teamwork model" en Team Performance Management, vol. 12, № 5-6, pp. 192-197. 